\title{
Measurement of Cerebro-Placental Doppler Ratio and Amniotic Fluid Index as a Predictor of Perinatal Outcome in Prolonged Pregnancy
}

\author{
Original \\ Article \\ Noha Hamed Abdelhamid Rabei, Mohamed Osama Taha', Mohamed Kamal \\ Etman $^{1}$, Omer Nasser Abbas Ali ${ }^{2}$ \\ ${ }^{I}$ Department of Obstetrics and Gynecology, Faculty of Medicine, Ain-Shams University, \\ 2Department of Obstetrics and Gynecology, Faculty of Medicine, Assiut University and Police \\ Academy Hospital, Egypt
}

\begin{abstract}
Background: The terms post-term, prolonged, postdates and post mature are often loosely used interchangeably to signify pregnancies that have exceeded a duration considered to be the upper limit of normal.

Objective: The aim of this work was to study the role of Doppler velocimetry of the umbilical and middle cerebral arteries represented by the cerebroplacental ratio and amniotic fluid volume in the prediction of adverse fetal outcome in post-term pregnancies.

Materials and Methods: This is a prospective case control study conducted at Ain-Shams University Teaching Hospital for Obstetrics and Gynecology, Egypt. The study included 50 pregnant patients who were divided into two groups; group1 included 25 pregnant ladies with gestational age of 41 weeks attending the casuality department in labour or in prodroma of labor. while, group 2 included 25 pregnant ladies with gestational age of 41 weeks not in labor and reaching the hospital for ANC, who were selected for termination based on the biophysical profile and poor Doppler indices or CTG changes. All patients were submitted to antenatal fetal surveillance tests including modified biophysical profile (MBPP) which consists of the non-stress test (NST), amniotic fluid index (AFI), and color Doppler velocimetry of fetoplacental and fetal vessels including MCA PI, UA PI, and CPR. The accuracy of cerebroplacental ratios (the middle cerebral artery PI divided by the umbilical artery PI) and amniotic fluid volume as a means of predicting intrauterine fetal distress and adverse perinatal outcome in post-term pregnancies.

Results: Cerebroplacental ratio showed the highest sensitivity (95\%) in comparison with other parameters, so it is a good test to reassure the obstetricians of the fetal well being. Prominent changes in AFI (i.e. $<50 \%$ increase or decrease) is not associated with adverse perinatal outcome irrespective of the rate of change provided that the final value remains $>5.0 \mathrm{~cm}$. A significant association with FHR decelerations and presence of meconium is proved to exist when AFI is $<5.0 \mathrm{~cm}$.

Conclusion: The addition of cerebral/umbilical ratios to antenatal surveillance protocols is expected to improve the perinatal outcome. It should be tried in the various high risk pregnancies whenever uteroplacental insufficiency is suspected.
\end{abstract}

Key Words: Doppler ultrasound, perinatal outcome, post-term, pregnancy

Received: 7 December 2019, Accepted: 22 January 2020

Corresponding Author: Omer Nasser Abbas Ali, Department of Obstetrics and Gynecology, Faculty of Medicine, Assiut University, Egypt, Tel.: 01025444402, E-mail: nomar1639@gmail.com

ISSN: 2090-7265, November 2020, Vol. 10, No. 4

\section{INTRODUCTION}

The terms post-term, prolonged, postdates and post mature are often loosely used interchangeably to signify pregnancies that have exceeded a duration considered to be the upper limit of normal ${ }^{[8]}$.

The standard internationally recommended definition of prolonged pregnancy accepted by both the World Health Organization (WHO) and the International Federation of Gynecology and Obstetrics (FIGO) is 42 completed weeks (294 days) or more from the first day of the last menstrual period (LMP), assuming valid dates and a regular 28 day cycle ${ }^{[19]}$. This definition was endorsed by the American College of Obstetricians and Gynecologists (1997) ${ }^{[9]}$.

Postdate pregnancies account about 5-10\% of the deliveries and are known to be associated with increased risk for perinatal complications ${ }^{[16]}$.

Incidence of subsequent post term birth increases from 10 to $27 \%$ if first birth was post term up to $39 \%$ if there had been two previous successive post term deliveries ${ }^{[2]}$. 
The mechanism of fetal complications associated with postdate pregnancy has attributed to progressive placental insufficiency, particularly in the presence of decreased amniotic fluid $^{[17]}$.

Placental dysfunction is considered to be the pathophysiologic event leading to intrapartum asphyxia and meconium aspiration. However, the principal mechanism leading to intrapartum fetal distress is umbilical cord compression due to oligohydramnios ${ }^{[10]}$.

Prepartum meconium aspiration is the potential danger in postdate pregnancy. Meconium staining of amniotic fluid serves as a primary ingredient in the lower APGAR scores and higher incidence of meconium aspiration syndrome and fetal distress encountered in the postdate group ${ }^{[4]}$.

Postdate infants, particularly those with macrosomia and post maturity, are at increased risk for hypoglycemia because post mature infants has decreased storage of glycogen and subcutaneous fat and also possibly an increased metabolic rate. It is important that postdate infants with macrosomia or signs of post maturity have glucose evaluations especially during the first 12 hours $^{[28]}$.

Postdate pregnancies are also associated with maternal risks namely discomfort, anxiety and increased cesarean delivery due to cephalopelvic disproportion. Also, there is a risk for cervical tears due to the fetal macrosomia ${ }^{[23]}$.

Ultrasound examination plays a very important role in the diagnosis of postdate pregnancies which is used for assessment of both gestational age and amniotic fluid volume. The amniotic fluid index (AFI) and biophysical profile (BPP) have both been used to assess fetal well-being ${ }^{[21]}$.

Doppler information may play a role in the surveillance of uncomplicated prolonged pregnancies. Data from reliable, well-constructed normal curves during this gestational age are lacking ${ }^{[25]}$.

A few studies had looked into the Doppler blood flow changes in postdated pregnancies and came up with varying results. Some demonstrated redistribution of blood flow in the fetal cerebral circulation in postdated pregnancies with adverse perinatal outcome, and such brain- sparing phenomenon is thought to result in oligohydramnios ${ }^{[27]}$.

However, others reported insignificant difference in Doppler indices for postdated pregnancies with and without oligohydramnios ${ }^{[16]}$.

Evaluation of the cerebral blood flow in the fetus has become an integrated part of the assessment of highrisk pregnancies. The middle cerebral artery (MCA) has been studied extensively, and its Doppler recordings are incorporated regularly into the management of fetuses at risk of developing placental compromise and fetal anemia $^{[11]}$

Combining the Doppler waveform analysis of the middle cerebral artery (MCA) with that of the umbilical artery (UA) by a common cerebro-placental ratio, i.e. the ratio of their pulsatility indices has been suggested as a useful clinical simplification ${ }^{[11]}$.

A low cerebroplacental ratio reflects redistribution of the cardiac output to the cerebral circulation and has been shown to improve accuracy in predicting adverse outcome compared with MCA or UA Doppler alone ${ }^{[32]}$.

\section{AIM OF THE WORK}

The aim of this study is to determine the role of Doppler velocimetry of the umbilical and middle cerebral arteries, represented by the cerebroplacental ratio, and amniotic fluid volume in the prediction of adverse fetal outcome in post-term pregnancies.

\section{PATIENTS AND METHODS}

This prospective cohort study included 50 pregnant patients admitted to Ain-Shams University Teaching Hospital for Obstetrics and Gynecology in the period starting from March 2016 to December 2019.

The patients were divided into two groups; group 1 included 25 pregnant ladies with gestational age of 41 weeks attending the casuality department in labour. While, group 2 included 25 pregnant ladies with gestational age of 41 weeks not in labor and reaching the hospital for ANC, who were selected for termination based on the biophysical profile and poor Doppler indices or CTG changes.

\section{Inclusion criteria:}

1- Age from 25-35 years old.

2- Singleton, viable fetus in the vertex presentation.

3- History of regular menstrual cycles.

4- Gestational age calculated from the first day of last menstrual period or by first-trimester or early secondtrimester ultrasound examination.(especially before 20 weeks)

\section{Exclusion criteria:}

1- Patients unsure of their dates.

2- Medical disorders with pregnancy as (hypertension, diabetes mellitus and Rhisoimmunization).

3- Prelabor rupture of membranes.

4- Polyhydramnios.

5- Chorioiamniotis 
6- Women with multiple pregnancies.

7- Congenital fetal anomalies.

8- Antepartum hemorrhage.

9- Previous cesarean section.

10- Fetal malpresentations.

Both groups were subjected to a detailed history including personal history, menstrual history, obstetric history, present history and past history. As well as physical examination including general, abdominal and pelvic examination to exclude those not fitting with the above listed criteria. Morover, investigations upon reaching the hospital for ANC.

All patients were subjected to transabdominal ultrasound and Doppler studies where the following was assessed :

A- Ultrasound and Doppler studies for:

1- Amniotic fluid index(AFI)

2- Gestational age determination

3- Fetal weight estimation

4- Middle cerebral artery pulsatility index (MCA-PI)

5- Umbilical artery pulsatility index(UA-PI)

6- Cerebro-placental ratio(CPR).

B- Cardiotocography (CTG) : All patients in labour were put under continuous electronic fetal monitoring, those not in labour were subjected to a non-stresstest.

\section{Technique of ultrasound and Doppler examination:}

Transabominal ultrasound performed to all patients while in a slightly tilted position with the head of the bed raised 30 degrees and with a small pillow under the right loin. The apparatus which used was the Voleson 730 ProV ultrasound machine with Doppler unit and convex linear transducer 3.5MHz.

Accordingly, together with the ultrasound findings and the Doppler readings:

Patients with non-reassuring fetal heart rate pattern on (CTG) and or the presence of low biophysical profile or altered Doppler indices (MCA-PI or UA-PI) were scheduled for termination whether thus induction of labour or cesarean section.

Patients where the decision was taken for conservation for good biophysical profile and good Doppler US (MCA, UA) were excluded from the study, but they were advised for repeated ultrasound and Doppler assessment until spontaneous labour or induction at 42 gestational weeks.

In patients where the decision for induction of labour was decided, when amniotomy is done, the color and amount of liquor was recorded. Birth weight, Apgar score at $1 \mathrm{~min}$ and $5 \mathrm{~min}$, gender and mode of delivery were noted.

Adverse pregnancy outcome was defined as the presence of one or more of the following conditions:
1- 5-min Apgar score is less than 7.

2- Transient tachypnea of the newborn(TTN).

3- Respiratory Distress syndrome.

4- Meconium aspiration syndrome.

5- Neonatal intensive care unit (NICU) admission.

\section{STATISTICAL ANALYSIS:}

Data were statistically described in terms of mean \pm standard deviation $( \pm \mathrm{SD})$, median and range, or frequencies (number of cases) and percentages when appropriate. $\mathrm{p}$ values less than 0.05 was considered statistically significant. All statistical calculations were done using computer programs SPSS (Statistical Package for the Social Science; SPSS Inc., Chicago, IL, USA) version 15 for Microsoft Windows.

\section{RESULTS}

A statistically significant difference between both groups as regard UA-PI, MCA-PI and CPR by using $\mathrm{P}$-value $(P>0.05)$ was shown in table 1.

The data represented in table 2 showed that cases with CS delivery had more adverse neonatal outcome compared with cases with vaginal delivery with statistically significant difference between both groups by using unpaired t-test $(P>0.05)$.

The data of table 3 showed that cases with CS delivery had a lower CPR and MCA (PI) compared to cases with VD with statistically significant difference in between by using $\mathrm{P}$ value $p<0.05$. On the other hand, there is no statistically significant difference between both groups as regard other markers.

A statistically highly significant positive correlation between AFI and MCA-PI, CPR, 5-min Apgar score $<7$ was oberved; while, negative correlation with CS for fetal distress, thick MSL and MAS. MCA-PI had a highly significant negative correlation with UA-PI, CS for fetal distress, thick MSL and MAS, while positive correlation with 5-min Apgar score $<7$ and UA $\mathrm{PH}<7.2$ and highly significant positive correlation with CPR (Tables 4 and 5).

There is a highly significant negative correlation between CPR and thick MSL and MAS and significant negative correlation with CS for fetal distress, while significant positive correlation with 5-min Apgar score $<7$.

On the other hand, UA-PI had highly significant inverse correlation with AFI and significant inverse correlation with CPR, while it has significant positive correlation with CS for fetal distress by using correlation coefficient rank test and no significant correlation with other variables (Figures 1, 2 and 3). 
Table 1: Comparison between both groups as regard Doppler indices

\begin{tabular}{lccc}
\hline & Group A & Group B & \\
& $\mathrm{N}=25$ & $\mathrm{~N}=25$ & \\
& & & \\
& & & \\
MCA-PI & $1.51+0.28$ & $1.58+0.21$ & $>0.05$ \\
Mean \pm S/D & $(0.98-1.78)$ & $(1-1.81)$ & $\mathrm{NS}$ \\
Range & & & \\
UA-PI & $0.75+0.10$ & $0.83+0.03$ & $>0.05$ \\
Mean \pm S/D & $(0.6-0.99)$ & $(0.65-1.02)$ & $\mathrm{NS}$ \\
Range & & & \\
CPR & & & \\
Mean $\pm \mathrm{S} / \mathrm{D}$ & $1.51 \pm 0.62$ & $1.58 \pm 0.63$ & $>0.05$ \\
& & & $\mathrm{NS}$ \\
Range & $(1.1-2.25)$ & $(1.27-2.9)$ & \\
\hline
\end{tabular}

MCA-PI: Middle cerebral artery pulsatility index; UA-PI: Umbilical artery pusatility index; CPR: Cerebral placental ratio $P$ values $=0,61,0.73,0.28$

Table 2: Relation between adverse fetal out come and mode of delivery

\begin{tabular}{lccc}
\hline & $\begin{array}{c}\text { VAGINAL } \\
\text { No=25 }\end{array}$ & $\begin{array}{c}\mathrm{C} / \mathrm{S} \\
\mathrm{No}=25\end{array}$ & $P$ \\
\hline Thick MSL & $8(10 \%)$ & $10(60 \%)$ & $<0.05 \mathrm{~S}$ \\
5 -min Apgar score $<7$ & 0 & $2(33 \%)$ & $<0.05 \mathrm{~S}$ \\
$\begin{array}{l}\text { Transient tachypnea of } \\
\text { the newborn (TTN) }\end{array}$ & $5(15 \%)$ & $7(80 \%)$ & $<0.05 \mathrm{~S}$ \\
$\begin{array}{l}\text { MAS } \\
\text { Respiratory Distress }\end{array}$ & $1(3 \%)$ & $1(16 \%)$ & $<0.05 \mathrm{~S}$ \\
$\begin{array}{l}\text { syndrome } \\
\text { NICU admission }\end{array}$ & $1(3 \%)$ & $3(50 \%)$ & $<0.05 \mathrm{~S}$ \\
$\begin{array}{l}\text { Total adverse } \\
\text { perinatal outcome }\end{array}$ & $5(13 \%)$ & $5(80 \%)$ & $<0.05 \mathrm{~S}$ \\
\hline
\end{tabular}

$\mathrm{MSL}=$ meconium stained liquor; $\mathrm{MAS}=$ meconium aspiration syndrome; $\mathrm{C} / \mathrm{S}=$ cesarean delivery; $\mathrm{NICU}=$ neonatal intensive care unit. $P$ values $=0.002,0.04,0.027,0.001,0.03,0.046,0.003$
Table 3: Relation between Doppler indices and AFI with mode of delivery

\begin{tabular}{lccc}
\hline \multicolumn{4}{c}{ MODE } \\
Variables & VD & CS & $P$ \\
& $1.8+1.5$ & $1.1+0.2$ & $<0.05 \mathrm{~S}$ \\
MCA-PI & $0.72+0.10$ & $0.76+0.17$ & $>0.05 \mathrm{NS}$ \\
UA-PI & $1.6+0.34$ & $1.3+0.2$ & $<0.05 \mathrm{~S}$ \\
CPR & $8.8+2.8$ & $8.3+3.6$ & $>0.05 \mathrm{NS}$ \\
AFI & & &
\end{tabular}

$P$ values $=0.12,0.029,0.37$

Table 4: Correlation between Doppler indices, AFI and fetal outcome

\begin{tabular}{lccccc}
\hline & $\begin{array}{c}\text { CS* for } \\
\text { fetal } \\
\text { distress }\end{array}$ & $\begin{array}{c}\text { Thick } \\
\text { MSL * }\end{array}$ & MAS * & $\begin{array}{c}\text { 5-min } \\
\text { Apgar } \\
\text { score } \\
<7\end{array}$ & $\begin{array}{c}\text { NICU* } \\
\text { admission }\end{array}$ \\
\hline AFI & -0.39 & $-0.88^{* *}$ & $-0.88^{* *}$ & 0.49 & $0.15-$ \\
MCA- & 0.77 & $0.34-$ & $0.43-$ & 0. & $0.33-$ \\
PI & & & & & \\
UA-PI & 0.33 & 0.15 & 0.13 & $0.05-$ & 0.14 \\
CPR & $0.33-$ & $0.72-$ & $0.62-$ & 0.38 & $0.32-$ \\
\hline
\end{tabular}

Table 5: Relation between Doppler indices and AFI

\begin{tabular}{lcccc}
\hline & AFI & MCA-PI & UA-PI & CPR \\
\hline AFI & - & $0.51^{* *}$ & $-0.62^{* *}$ & 0.63 \\
MCA-PI & $0.51^{* *}$ & - & $-0.39^{*}$ & $0.88^{*}$ \\
UA-PI & $-0.62^{* *}$ & $-0.39^{*}$ & - & -0.39 \\
CPR & $0.63^{* *}$ & $0.88^{* *}$ & $-0.39^{*}$ & - \\
\hline
\end{tabular}

$\mathrm{MSL}=$ meconium stained liqour. MAS $=$ meconium aspiration syndrome.

$\mathrm{CS}=$ cesarean section. $\quad$ NICU $=$ neonatal intensive care unit.

$\mathrm{UA}=$ umbilical artery. ${ }^{*} p<0.05=S * * p<0.001=H S$ 


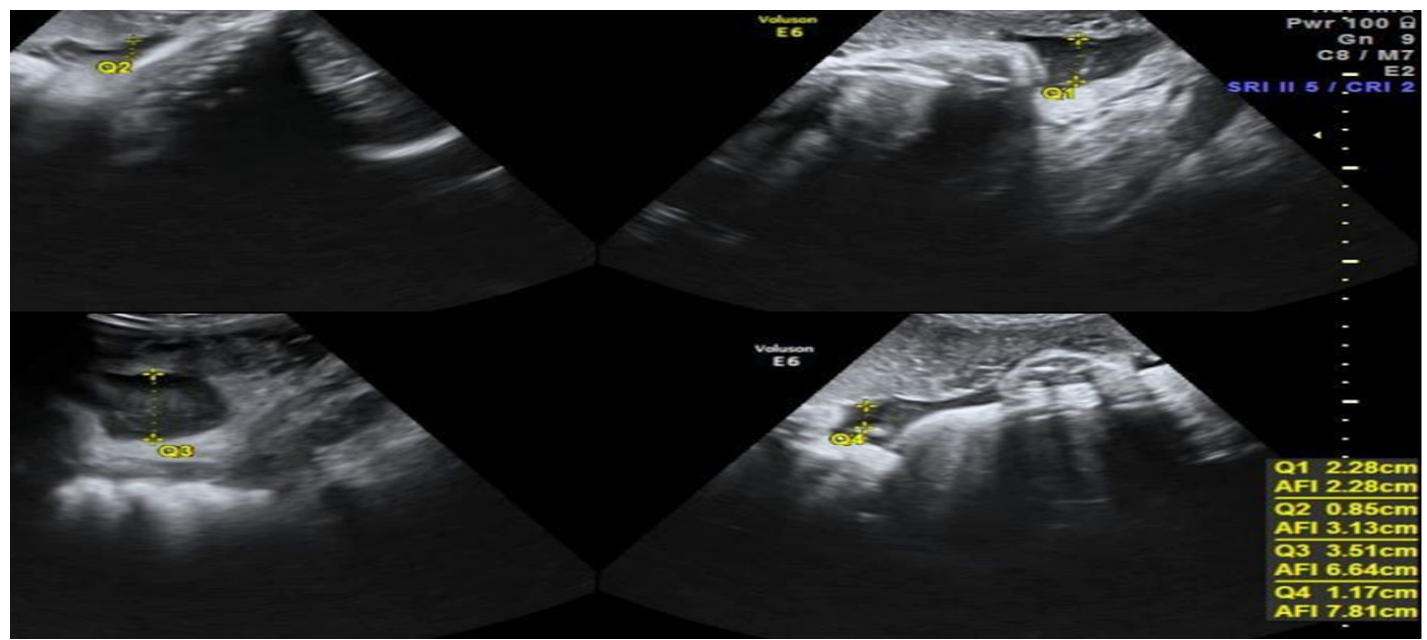

Fig. 1: Measurements of the largest vertical pocket of fluid in each of four quadrants of the uterus

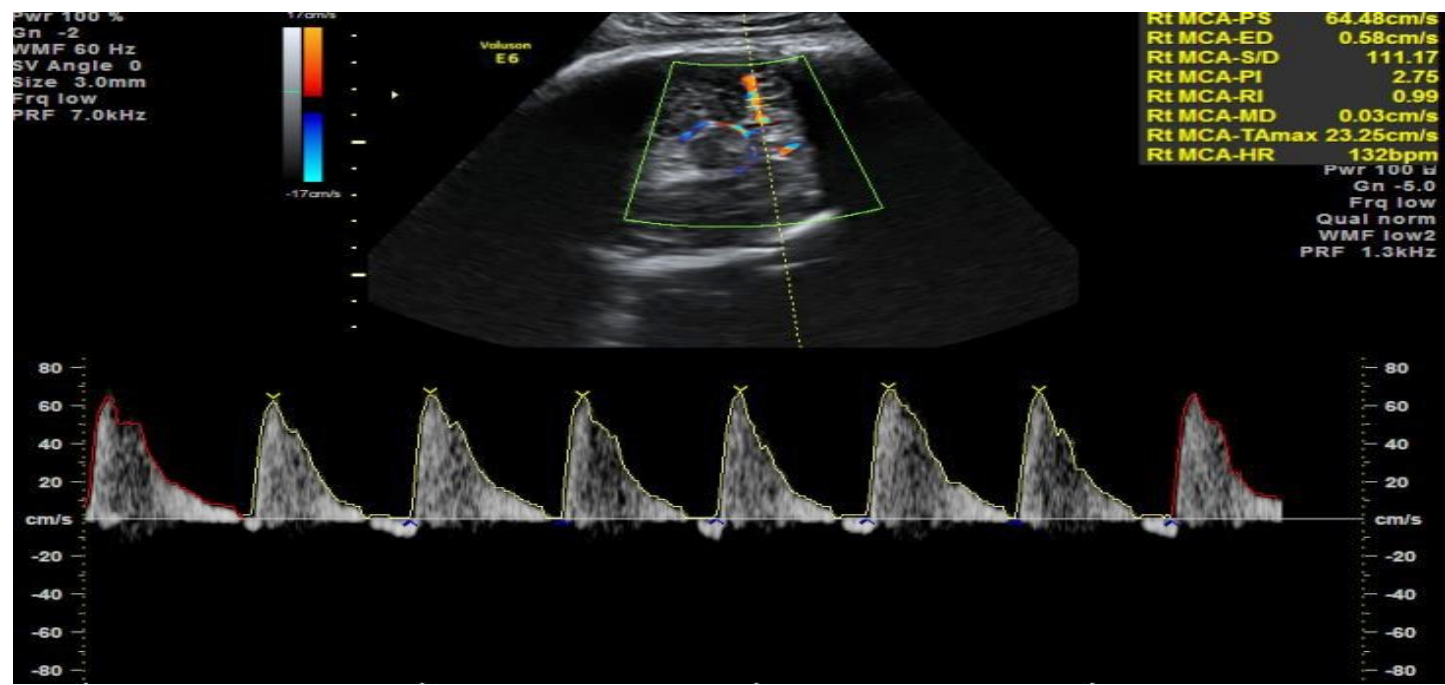

Fig. 2: Three consecutive MCA wave forms from which Middle cerebral artery pulsatility index (MCA-PI) is measured

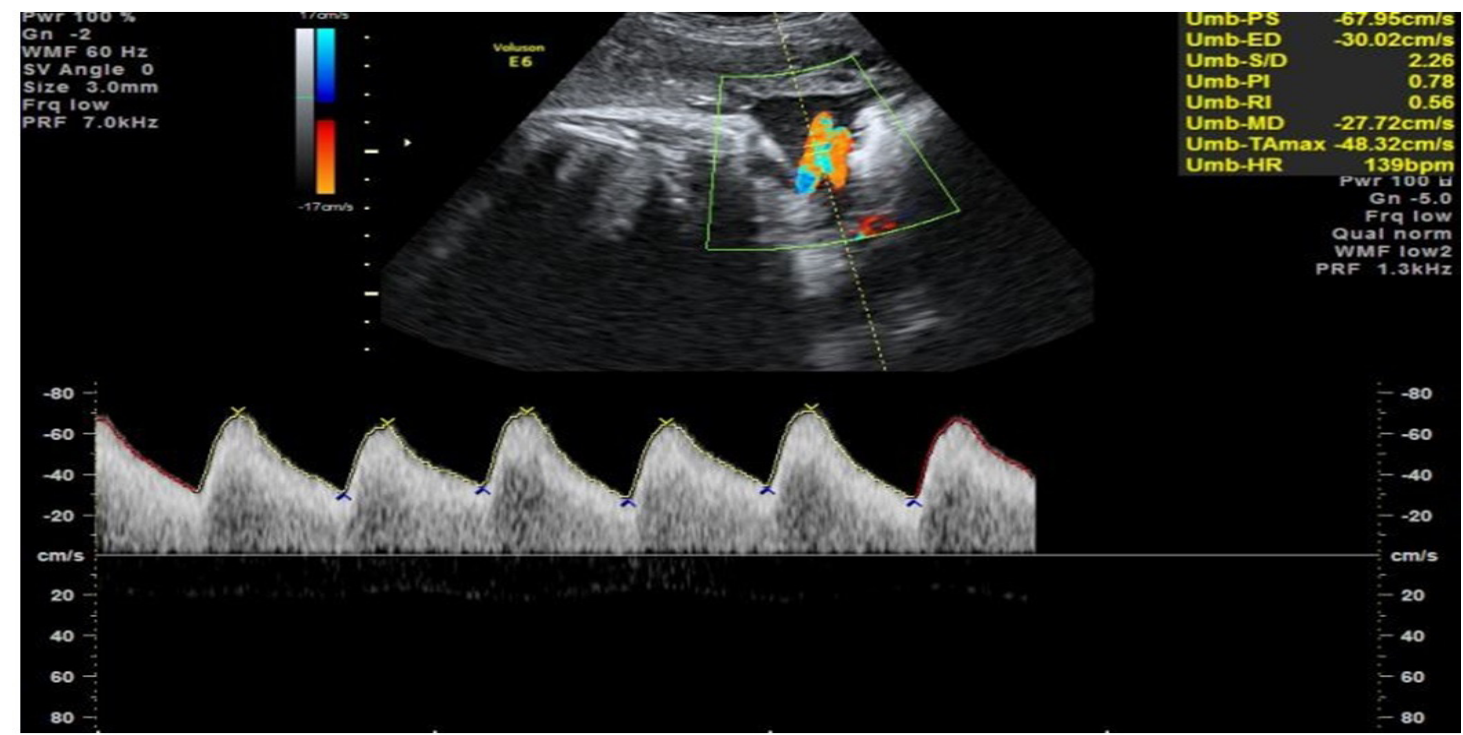

Fig. 3: Three consecutive UA wave forms from which Umbilical artery pulsatility index (UA-PI) is measured. 


\section{DISCUSSION}

Post-term pregnancies account for about 5-10\% of the deliveries and are known to be associated with increased risk for perinatal complications ${ }^{[16]}$.

It has been postulated that placental aging with increasing uteroplacental insufficiency is responsible for the morbidity and mortality in post-term pregnancy ${ }^{[25]}$.

Evaluation of fetoplacental circulation with Doppler ultra- sonography uses color flow imaging for detection of any alteration in cerebral circulation before changes in fetal heart rate pattern occurs by hypoxia. Therefore, cerebroplacental ratio has been introduced as an indicator of brain-sparing effect, which is one of the main fetal mechanisms to respond to chronic hypoxia ${ }^{[1]}$.

The intrapartum management of prolonged pregnancies is a unique challenge to the obstetrician, as the perinatal outcome is adverse after 40 completed weeks. In such cases, intrapartum asphyxia and meconium aspiration are associated with almost three fourths of all perinatal deaths ${ }^{[10]}$.

The aim of this study is to determine the role of Doppler velocimetry of the umbilical and middle cerebral arteries, represented by the cerebroplacental ratio, and amniotic fluid volume in the prediction of adverse fetal outcome in post-term pregnancies.

Thus we can evaluate the efficacy of these parameters in the assessment of intra partum fetal well-being and prediction of meconium aspiration and (MSL) meconium-stained liquor.

The present study included 50 pregnant ladies who were divided into two groups; group A including 25 pregnant ladies with gestational age of 41 weeks attending the causality department in labour and group B including 25 pregnant ladies with gestational age 41 weeks not in labor and reaching the hospital for ANC, who were selected for termination based on the biophysical profile and poor Doppler indices or CTG changes.

In the present study, regarding maternal age, body weight and parity, and the relation of these factors with prolonged pregnancy was found to be statistically nonsignificant among both groups.

However, Crowley ${ }^{[7]}$ found that prolonged pregnancy is increased in first pregnancies, but is not related to maternal age and the median duration of pregnancy is 2 days longer in nulliparas compared with multiparas.
As regards mode of delivery, $67.5 \%$ of cases in group A and $77.5 \%$ of cases in group B delivered vaginally. Indication for $\mathrm{CS}$ included fetal distress $(20 \%)$, failed induction $(6.7 \%)$ and failure of progress $(30 \%)$.

Luckas et al. ${ }^{[19]}$ showed that cesarean section was significantly more common in women with postterm pregnancy. The increase was equally distributed between Cesarean sections performed for failure to progress in labor and fetal distress. However; they reported that the possibility of bias in management arising out of the knowledge that a pregnancy is postterm cannot be excluded as a factor in the increase in cesarean section rates.

In the present study, thick meconium stained liquor, was present in about $(20 \%)$ of cases of both group. These results agree with study by Lam et al. ${ }^{[16]}$ that found a statistically significant increased rate of thick MSL in a homogenous group of Uncomplicated postdated pregnancies beyond 41 weeks.

Caughey et al. $\cdot^{[5]}$ conducted a retrospective study on low-risk, post term, cephalic, and singleton births and the incidence of adverse neonatal mortality outcomes at 40, 41 and 42 weeks' gestation was examined in a multivariate analysis and compared with the rates in pregnancies that were deliveredat 39 weeks of gestation, after controlling for maternal demographics, length of labor, induction, mode of delivery, and birth weight (except macrosomia). Compared with the outcome at 39 weeks' gestation, the relative risk of meconium aspiration increased significantly from 2.18 at 40 weeks to 3.35 at 41 weeks and 4.09 at 42 weeks. A composite outcome of severe neonatal complications including meconium aspiration syndrome increased from a relative risk of 1.47 at 40 weeks to 2.04 at 41 weeks to 2.37 at 42 weeks.

In the current study, 5-min Apgar score less than 7 showed no statistically significant between the two groups.

In the present study, $26.7 \%$ of neonates were admitted in NICU mainly due to low 5-min Apgar score, thick MSL. These results agree with study by Lam et al. (2005a) noticing that indications for admission to NICU were mainly for thick MSL with a few for maternal group B streptococcal colonization and low first-minute Apgar score.

Adverse perinatal outcome according to present study included fetal distress according to CTG, thick meconium stained liquor, transient tachypnea of the newborn (TTN), 5-min Apgar score less than 7 or NICU admission due to meconium aspiration syndrome or Respiratory Distress Syndrome (RDS). 
In the current study, there were neither structural abnormalities nor perinatal death encountered in this study. It is believed that the absence of perinatal death is due to the use of the recent methods of monitoring postdate fetuses, such as CTG and Doppler studies.

As regards the AFI in the present study, the mean values of AFI were $8.9+1.58$ in normal perinatal outcome and $5.9+2.1$ in adverse perinatal outcomes with a statistically significant difference between both groups. There was a significant relation between lower AFI and thick meconium stained liquor. So AFI was considered the most sensitive predictor of thick MSL and MAS.

Lam et $a l^{[17]}$ reported that although overall AFI correlated with thick MSL and the need for intervention for fetal distress, its sensitivity and specificity are limited, in post date pregnancies.

The results of the present study agree with study by Chauhan et al. ${ }^{[6]}$ that concluded that there was some association between oligohydramnios and an increased risk of cesarean section for non- reassuring fetal heart rate patterns and low Apgar scores. Similarly, Locatelli et al. ${ }^{[18]}$ that measured AFI twice weekly from 40 weeks until delivery.

A composite adverse outcome of fetal death, $5 \mathrm{~min}$ Apgar $<7$, umbilical artery $\mathrm{pH}$ of $<7$, Cesarean section for fetal distress, occurred in $19.8 \%$ of those with an AFI of $<5$ compared with $10.7 \%$ of those with an AFI of $>5$ with highly significant difference $(P=0.001)$.

Also, Pasquini et al. ${ }^{[26]}$ found the incidence of fetal distress and variable deceleration was significantly higher in the group with $\mathrm{AFI}=$ or $<5$. Also there was positive correlation between AFI and APGAR score and negative correlation between AFI and CS for fetal distress, thick meconium stained liquor and meconium aspiration syndrome. In the present study, the best cutoff value of AFI to predict adverse neonatal outcome above or equal 5 .

In the present study, the mean values of UA-PI were 0.75 in group $\mathrm{A}$ and 0.83 in group $\mathrm{B}$ with no statistically significant difference between both groups. These results agree with the study by Baschat and Gembruch ${ }^{[3]}$ that reported that the mean value of UA-PI in postdated group was 0.75 .

In the current study, UA-PI values did not show a statistically significant difference between normal and adverse perinaqtal outcome. These results agree with study by Tasic et al. ${ }^{[30]}$ that noticed that normal values of Doppler velocity indices in uteroplacenta lcirculation confirm the assumption that in pregnancy after $40^{\text {th }}$ gestation week problems arise not from the uteroplacental unit, but rather from changes in fetoplacental circulation. Normal values of Doppler indices in uterine arteries, umbilical and middle cerebral artery of fetus are found in pregnancy after $40^{\text {th }}$ week of gestation with unsatisfactory perinatal outcome. The results of their study suggested that unsatisfactory perinatal outcome is possible in postdate pregnancy, even if there is no placental insufficiency. This finding also shows that bad perinatal outcome is under the influence of some other variables that are not connected with prolonged pregnancy.

Similarly, Stokes et al. ${ }^{[29]}$ found that UA Doppler flow velocity wave forms from pregnancies associated with fetal compromise and abnormal neonatal outcome were similar to those from pregnancies in which the outcome was normal. They concluded that Doppler flow velocity waveform analysis is unlikely to be of benefit in the routine assessment of the postdate pregnancy. Also in this study, there was no role of UAPI in prediction of thick meconium stained liquor as UA-PI in cases with negative MSL was $0.74+0.14$ and in cases with positive MSL was $0.74+0.09$ with no statistically significant difference in between.

UA-PI had a significant inverse correlation with MCA-PI, CPR and AFI, while significant positive correlation with CS for fetal distress, Malcus et al. ${ }^{[22]}$. In a prospective study during 1 year, 102 women with prolonged pregnancies (more than 294 completed gestational days) were followed with serial pulsed Doppler blood flow examinations every $2^{\text {nd }}$ day, either to spontaneous onset of labor $(n=82)$ or to induction of labor due to subsequently occurring complications $(n=20)$, showed that flow velocity waveforms in the UA didn't change compared with values at term and that abnormal flow velocity had no significant relationship with fetal asphyxia, indicating that the placenta didn't alter fetal blood flow. These authors suggested that structural changes on the maternal or fetal side of the placenta are not sufficiently pronounced to alter flow resistance during prolonged pregnancy.

These observations could be explained by the fact that UA velocimetry is a test of placental function that does not always directly reflect fetal status. Similarly, Lam et al. ${ }^{[16]}$ found UA Doppler measurement revealed neither a sensitive marker to reflect liquor volume nor the perinatal outcome in postdated pregnancies.

In the present study, it was found that at a cutoff value of $\geq 0.89$, UA- PI had $50 \%$ sensitivity and $55 \%$ specificity in prediction of adverse fetal outcome. Also Fischer et al..$^{[13]}$ suggested a similar cutoff value of 0.82 to UA-PI. 
In the present study, the mean values of MCAPI were 1.51 in group A and 1.58 in group B with no statistically significant difference between both groups. These results agree with the study by Figueras et al. ${ }^{[12]}$ that reported that the mean value of MCA-PI in postdate group was1.45.

Normal perinatal outcome was associated with higher MCA-PI 1.3+0.25 compared to adverse perinatal outcomes $1.08+0.29$ with statistically significant difference between both groups. Cases with CS delivery had lower MCA-PI compared to cases with VD.

In this study, MCA-PI appeared to be a more useful tool in predicting passage of thick meconium stained liquor as cases with positive MSL had a lower MCA-PI1 2+0.3 compared to cases without MSL $1.8+1.3$ with statistically significant difference.

The MCA-PI had a highly significant positive correlation with CPR and AFI and significant positive correlation with 5-min Apgar score, while highly significant negative correlation with UA-PI, CS for fetal distress, thick MSL and MAS.

In the present study, regarding MCA-PI the best cut-off value for MCA-PI was $>1.1$ for good fetal outcome. In contrast, Ott et al. ${ }^{[24]}$ found a cutoff value of 1 for the MCA-PI.

Also, Usha et al. ${ }^{[31]}$ discovered a non significant statistical difference in umbilical artery Doppler or middle cerebral artery (PI) alone in predicting adverse perinatal outcome. They attributed this to that in uncomplicated postdate pregnancies; the mechanism of fetal compromise is perhaps due to decrease in the flow of nutrients across the placenta and decrease in the efficiency of utilization of nutrients by the placenta and the fetus. These changes are not reflected in increase in resistance in the blood vessels of placenta or fetus. This may explain the absence of any significant statistical difference in umbilical artery PI but in this study MCA PI value had significant statistical difference between normal and adverse perinatal outcome.

In the present study, the mean values of CPR were 1.51 in group $\mathrm{A}$ and 1.58 in group $\mathrm{B}$ with no statistically significant difference between both groups. These results agree with the study by Palacio et al. ${ }^{[25]}$ that reported that the mean value of CPR in postdate group ranged from 1.46 to 1.43 .

AS regards CPR, normal perinatal outcome was associated with a high mean CPR of $2.5+0.6$ while adverse perinatal outcome associated with a lower
CPR of $1.7+0.6$ and this difference was statistically significant. Also, cases with CS delivery had a lower mean CPR 1.3+0.2 compared to cases with VD $1.6+0.34$.

The CPR had strong positive correlation with MCA-PI and AFI and moderate positive correlation with 5-min Apgar score, while there was a strong negative correlation between CPR and thick meconium liquor (TML), moderate negative correlation with meconium aspiration syndrome (MAS) and mild negative with the need for CS due to fetal distress.

In the current study, the best cutoff of the CPR is 1.4 where $\mathrm{CPR}>1.4$ was considered normal and $\leq 1.4$ was considered abnormal. These results agree with Yoshimura et al. ${ }^{[33]}$. That selected a cutoff value for CPR of 1.4 based on extensive results.

In the present study, the sensitivity of CPR in prediction of adverse perinatal outcome was 95\%, its specificity $=50 \%$ with a $90 \%$ NPV. While UAPI showed a sensitivity of $50 \%$, specificity of $55 \%$, PPV of $60 \%$ and NPV $=54 \%$. The MCA-PI alone showed a higher specificity of $80 \%$. The sensitivity of AFI to predict prognosis of fetal outcome in this study was $40 \%$. Its specificity was $80 \%$ with PPV of $78 \%$, NPV of $50 \%$. On the other hand, combination of different markers improved both sensitivity and specificity.

These results agree with Baschat and Gembruch $^{[3]}$ where the PI was determined in the midportion of the umbilical artery and the mid or distal segment of the middle cerebral. The CPR offered the advantage of detecting redistribution of blood flow due to two potential mechanisms ; either forced centralization an increase of the placental resistance or increase in the cerebral blood flow due to a brainsparing effect, accordingly CPR was considered a better predictor of fetal compromise than either vessel considered alone. Similarly Hershkovitz et al. ${ }^{[15]}$ and Vergani et al. ${ }^{[32]}$ support these results regarding CPR being better predictor of fetal compromise than neither MCA-PI nor UA-PI alone.

However, Palacio et al. ${ }^{[25]}$ in their study on UA PI, MCA PI and CU ratio in postdated pregnancies concluded that Doppler information may play a role in differentiating postdated pregnancies which may be followed by expectant management from those in whom induction is a better option. However, low positive predictive value of $\mathrm{CU}$ ratio can result in missing cases with fetal jeopardy and high false positive rate can result in undue concern, expensive tests and unnecessary interference. In comparison to 
this, CPR showed a low PPV of $53 \%$ in the present study, however a high NPV of $90 \%$.

On the other hand, Hassan ${ }^{[14]}$ found that the sensitivity of AFI was $63.2 \%$ and its specificity was $83 \%$ which is similar to the specificity of AFI in the present study of $80 \%$. And found that AFI is a reliable fetal surveillance test. It may allow the conservative approach till 42 weeks to reduce the caesarean section rate due to failed induction of labour as well as perinatal morbidity and mortality.

In the present study, CPR showed the highest sensitivity (95\%) in comparison with other parameters followed by UA-PI $(50 \%)$. On the other hand, MCA-PI and AFI showed the highest specificity of $(80 \%)$ to each one. MCA-PI showed the highest PPV $(83 \%)$ while CPR had the highest NPV of $(90 \%)$ to adverse fetal outcome, the sensitivity of AFI to predict adverse perinatal outcome in this study was $(40 \%)$. Its specificity was $(80 \%)$, PPV was $(78 \%)$, and NPV was $(50 \%)$ but combination of all parameters was shown to improve both sensitivity and prediction.

\section{CONCLUSION}

In the surveillance of the uncomplicated prolonged pregnancy, Doppler information plays a role in differentiating which pregnancies may be followed by expectant management or determines whether induction is a better option.

When oligohydramnios is present in postdate pregnancy, there is a significantly greater likelihood of meconium staining of amniotic fluid. So, the post-term fetuses maybe at higher risk for meconium aspiration

Cerebroplacental ratio showed that the highest sensitivity (95\%) in prediction of adverse perinatal outcome comparison with other parameters, so it is a trustable test to reassure the obstetricians of the fetal wellbeing.

\section{CONFLICT OF INTEREST}

There are no conflicts of interests.

\section{REFERENCES}

1. Bahado-Singer RO, Oz AU, HsuC, et al (2000): Middle cerebral artery Doppler velocimetric deceleration angel as a predictor of fetal anemia in Rh-allo immunized fetuses without hydrops. Am J Obstst \& Gynecol; 183: 746

2. Bakketeig LS, Bergsjo P and Walther M (2000): Postterm pregnancy: magnitude of the problem. Effective care in pregnancy and child birth. Oxford University: 765-775.
3. Baschat A and Gembruch U (2003): The cerebroplacental ratio revisited. Ultrasound Obstet Gynecol; 21: 124-127.

4. Boisserlier P, Dame C, Finne PH and Guettier $X$ (2001): Prolonged pregnancy. Review of literature. J Gynecol Obstet Biol Repord (Paris); 24 (7): 739-746.

5. Caughey $\mathrm{AB}$, Washington $\mathrm{AE}$ and Laros $\mathrm{RK}$ (2005): Neonatal complications of term pregnancy: rates by gestational age increase in a continuous, not threshold, fashion. Am J Obstet Gynecol; 192: 185-190.

6. Chauhan SP, Sanderson M, Hendrix NW, Magann EF and Devoe LD (1999): Perinatal outcome and amniotic fluid index in the antepartum and intrapartum periods: a meta-analysis. Am J Obstet Gynecol; 181: 1473-1478.

7. Crowley P (2005): Prolonged pregnancy. In: Dewhurst's Textbook of Obstetrics and Gynecology, Edmonds DK (editor), 7th ed. Blackwell publishing; 192-204.

8. Cunningham FG, Hauth JC, Leveno KJ, Gilstrap L, Bloom SL and Wenstrom KD (2017): Antepartum assessment. In: William Obstetrics. Cunningham FG, Hauth JC, Leveno KJ, Gilstrap L, Bloom SL and Wenstrom KD (eds). 22nd ed. New York: McGraw-Hill;373-387.

9. Cunningham FG, Hauth JC, Leveno KJ, Gilstrap L, Bloom SL and Wenstrom KD (2015): Intrapartum assessment. In: Williams Obstetrics, Cunningham FG, Hauth JC, Leveno KJ, Gilstrap L, Bloom SL and Wenstrom KD (eds). 22nd ed. New York: McGraw-Hill;443-471.

10. Dasari P, Niveditta G and Raghavan S (2007): The maximal vertical pocket and amniotic fluid index in predicting fetal distress in prolonged pregnancy. Int J Gynecol Obstet; 96: 89-93.

11. Ebbing C, Rasmusssen S and Kiserud T (2007): Middle cerebral artery blood flow velocities and pulsitility index and the cerebroplacental pulsitility ratio: longitudinal reference ranges and terms for serial measurements. Ultrasound Obstet Gynecol; 30: 287-296.

12. Figueras $\mathrm{F}$, Lanna $\mathrm{M}$, Palacio $\mathrm{M}$, Zamora L, Puerto B, Coll O, Cararach V and Vanrell A (2004): Middle cerebral artery Doppler indices at different sites: prediction of umbilical cord gases in prolonged pregnancies. Ultrasound Obstet Gynecol; 24: 529-533. 
13. Fischer RL, Khulman KA, Depp R and Wapner RJ (1991): Doppler evaluation of umbilical and uterine arcuate in the postdate pregnancies. Obstet Gynecol; 78 (3): 363-368.

14. Hassan AA (2005): The role of amniotic fluid index in the management of postdate pregnancy. $\mathrm{J}$ Coll Physicians Surg Pak; 15(2): 85-88.

15. Hershkovitz R, Kingdom JCP, Geary M, Rodeck CH (2000): Fetal cerebral blood flow redistribution in late gestation: identification of compromise in small fetuses with normal umbilical artery Doppler. Ultrasound Obstet Gynecol; 15: 209-212.

16. Lam H, Leung WC, Lee CP and Lao TT (2005a): The use of fetal Doppler cerebroplacental blood flow and amniotic fluid volume measurement in the surveillance of postdated pregnancies. Acta Obstet Gynecol Scand; 84: 844-848.

17. Lam H, Leung WC, Lee CP and Lao TT (2006): Amniotc fluid volume at 41 weeks and infant outcome. J Reprod Med; 51 (6): 484-488.

18. Locatelli A, Zagarell A, Toso L, Assi F, Ghidini A \& Biffi A (2004): Serial assessment of amniotic fluid index in uncomplicated term pregnancies: prognostic value of amniotic fluid reduction. J Matern Fetal Neonat Medical; 15: 233-236.

19. Luckas M (2004): Prolonged pregnancy. In: Obstetrics and Gynecology. Lesley DM, Baker PN et al., (eds). 1st ed. London press; 272-275.

20. Luckas M, Buckett W and Alfirevic Z (1998): Comparison of outcomes in uncomplicated term and post-term pregnancyfollowing spontaneous labor. J Perinat Med; 26: 475-9.

21. Magnann EF, Doherty DA and Chauhan SP (2004): How will do the amniotic fluid index and single deepest pocket indices (Below the $3^{\text {rd }} \& 5$ th and above the 95th and 97th percentiles). Am J Obstet \& Gynecol; 190:164-169.

22. Malcus P, Marsal K and Persson PH (1991): Fetal and uteroplacental blood flow in prolonged pregnancies, a clinical study. Ultrasound Obstet Gynecol; 1: 40-45.

23. Olesen AW, Westergaard JG and Olsen J (2003): Perinatal and maternal complication related to postterm delivery: a national register- based study. 1978-1993. Am J Obstet Gynecol; 189: 222-227.
24. Ott WJ, Mora G and Arias F (1998): Comparison of the modified biophysical profile to a new biophysical profile incorporating the middle cerebral artery velocity flow systolic/diastolic ratio. Am J Obstet Gynecol; 178: 1346-53.

25. Palacio M, Figueras F, Zamora L, Jimenez JM, Puerto B, Coll O, Cararach and Vanrell JA (2004): Reference ranges for umbilical and middle cerebral artery pulsatility index and cerebroplacental ratio in prolonged pregnancies. Ultrasound Obstet Gynecol; 24:647-653.

26. Pasquini L, Nasto R, Mie ME, Giuliani B and Periti E (2003): Amniotic fluid analysis as a screening test in term and postterm pregnancy. Minerva gynecol; 55(1): 69-73.

27. Selam B, Koksal R and Ozcan T (2000): Fetal arterial and venous Doppler parameters in the interpretation of oligohydramnios in post-term pregnancies. Ultrasound Obstet Gynecol; 15: 403406.

28. Sims ME, Machelln H and Waltter FJ (2001): Neonatal morbidity and mortality long-term outcome of postdate infants. Clin Obstet Gynecol; 32; (2): 285-293.

29. Stokes HJ, Roberts RV and Newnham JP (2008): Doppler flow velocity waveform analysis in postdate pregnancies. Aust N Z J Obstet Gynecol; 31 (1): 27-30

30. Tasic M, Lilic V, Milosevic J, Antic V and Stefanovic M (2007): Placental insufficiency in pregnancy after 40th week of gestation. Acta Medica Medianae; 46: 26-29.

31. Usha G, Suhasini C, Narula MK (2006): Value of middle cerebral artery to umbilical artery ratio by doppler velocimetry in pregnancies beyond term $\mathbf{J}$ Obstet Gynecol Indi.. 56 (1):37-40.

32. Vergani $\mathrm{P}$, Roncalgia N, Locatelli A, Andreotti C, Crippa I, Pezzullo JC and Ghidini A (2005): Antenatal predictors of neonatal outcome in fetal growth restriction with absent end-diastolic flow in the umbilical artery. Am J Gynecol; 193: 12131218.

33. Yoshimura S, Masuzaki H, Muira K, et al. (1998): Fetal blood flow redistribution in term intrauterine growth retardation (IUGR) and postnatal growth. International Journal of Gynecology and Obstetrics; 9: 3-8. 